\title{
Lyapunov Instability and Collective Tangent Space Dynamics of Fluids
}

\author{
Harald A. Posch and Ch. Forster \\ Institut für Experimentalphysik, Universität Wien, \\ Boltzmanngasse 5, A-1090 Vienna, Austria
}

\begin{abstract}
The phase space trajectories of many body systems charateristic of isimple fluids are highly unstable. We quantify this instability by a set of Lyapunov exponents, which are the rates of exponential divergence, or convergence, of infinitesimal perturbations along selected directions in phase space. It is demonstrated that the perturbation associated with the maximum Lyapunov exponent is localized in space. This localization persists in the large-particle limit, regardless of the interaction potential. The perturbations belonging to the smallest positive exponents, however, are sensitive to the potential. For hard particles they form well-defined long-wavelength modes. The modes could not be observed for systems interacting with a soft potential due to surprisingly large fluctuations of the local exponents.
\end{abstract}

\section{Lyapunov spectra}

Recently, molecular dynamics simulations have been used to study many body systems representing simple fluids or solids from the point of view of dynamical system theory. Due to the convex dispersive surface of the atoms, the phase trajectory of such systems is highly unstable and leads to an exponential growth, or decay, of small (infintesimal) perturbationis of an initial state along specified directions in phase space. This so-called Lyapunov instability is described by a set of rate constants, the Lyapunov exponents $\left\{\lambda_{l}, l=1, \ldots, D\right\}$, to which we refer as the Lyapunov spectrum. Conventionally, the exponents are taken to be ordered by size, $\lambda_{l} \geq \lambda_{l+1}$. There are altogether $D=2 d N$ exponents, where $d$ is the dimension of space, $\mathrm{N}$ is the number of particles, and $D$ is the dimension of the phase space. For fluids in nonequilibrium steady states close links between the Lyapunov spectrum and macroscopic dynamical properties, such as transport coefficients, irreversible entropy production, and the Second Law of thermodynamcis have been established [1-4]. This important result provided the motivation for us to examine the spatial structure of the various perturbed states associated with the various exponents. Here we present some of our results for two simple many-body systems representing dense two-dimensional fluids in thermodynmic equilibrium. The first model consists of $N$ hard disks (HD) interacting with hard elastic collisions, the second of $N$ soft disks interacting with a purely repulsive Weeks-Chandler-Anderson (WCA) potential. 
The instantaneous state of a planar particle system is given by the $4 \mathrm{~N}$ dimensional phase space vector $\boldsymbol{\Gamma}=\left\{\mathbf{r}_{i}, \mathbf{p}_{i}, ; i=1, \ldots, N\right\}$, where $\mathbf{r}_{i}$ and $\mathbf{p}_{i}$ denote the respective position and linear momentum of molecule $i$. An infinitesimal perturbation $\delta \boldsymbol{\Gamma}=\left\{\delta \mathbf{r}_{i}, \delta \mathbf{p}_{i} ; i=1, \ldots, N\right\}$ evolves according to motione equations obtained by linearizing the equations of motion for $\boldsymbol{\Gamma}(t)$. For ergodic systems there exist $D=4 N$ orthonormal initial vectors $\left\{\delta \boldsymbol{\Gamma}_{l}(0) ; l=1, \ldots, 4 N\right\}$ in tangent space, such that the Lyapunov exponents

$$
\lambda_{l}=\lim _{t \rightarrow \infty} \frac{1}{t} \ln \frac{\left|\delta \boldsymbol{\Gamma}_{l}(t)\right|}{\left|\delta \boldsymbol{\Gamma}_{l}(0)\right|}, l=1, \ldots, 4 N .
$$

exist and are independent of the initial state. Geometrically, the Lyapunov spectrum describes the stretching and contraction along linearly-independent phase space directions of an infinitesimal hypersphere co-moving with the flow. For equilibrium systems the symplectic nature of the motion equations assures the conjugate pairing rule to hold [5]: the exponents appear in pairs, $\lambda_{l}=\lambda_{4 N+1-l}=$ 0 , so that only half of the spectrum $\left\{\lambda_{1 \leq l \leq 2 N}\right\}$ needs to be cqaluclated. The sum of all Lyapunov exponents vanishes, which, according to Liouville's theorem, expresses the fact that the phase volume is strictly conserved for Hamiltonian systems. Six if the exponents $\left\{\lambda_{2 N-2 \leq l \leq 2 N+3}\right\}$ always vanish as a consequence of the conservation of energy, momentum, and center of mass, and of the nonexponential time evolution of a perturbation vector parallel to the phase flow.

For the computation of a complete spectrum a variant of a classical algorithm by Benettin et al. and Shimada et al. is used $[6,7]$. It follows the time evolution of the reference trajectory and of an orthonormal set of tangent vectors $\left\{\delta \boldsymbol{\Gamma}_{l}(t) ; l=\right.$ $1, \ldots, 4 N\}$, where the latter is periodically re-orthonormalized with a GramSchmidt (GS) procedure after consecutive time intercals $\Delta t_{G S}$. The Lyapunov exponents are determined from the time-averaged renormalization factors. For the hard disk systems the free evolution of the particles is interrupted by hard elastic collisions and a linearized collision map needs to be calculated as was demonstrated by Dellago et al. [9]. Although we make use of the conjugate pairing symmetry and compute only the positive branch of the spectrum, we are presently restricted to about 1000 particles by our available computer resources.

For our numerical work reduced units are used. In the case of the WeeksChendler-Anderson interaction potential,

$$
\phi(r)=\left\{\begin{array}{lr}
4 \epsilon\left[(\sigma / r)^{12}-(\sigma / r)^{6}\right]+\epsilon, & r<2^{1 / 6} \sigma \\
0, & r \geq 2^{1 / 6} \sigma .
\end{array},\right.
$$

the particle mass $m$, the particle diameter $\sigma$, and the time $\left(m \sigma^{2} / \epsilon\right)^{1 / 2}$ are unity. In this work we restrict our discussion to a thermodynamic state with a total energy per particle, $E / N$, also equal to unity. For the hard-disk fluid $\left(N m \sigma^{2} / K\right)^{1 / 2}$ is the unit of time, where $K$ is the total kinetic energy, which is equal to the total energy $E$ of the system. There is no potential energy in this case. The reduced temperature $T=K / N$, where Boltzmann's constant is also taken unity. In the following, Lyapunov exponents for the two model fluids will be compared for equal temperatures (and not for equal total energy). This requires a 
rescaling of the hard-disk exponents by a factor of $\sqrt{K_{W C A} / K_{H D}}$ to account for the difference in temperature. All our simulations are for a reduced density $\rho \equiv N / V=0.7$, where the simulation box is a square with a volume $V=L^{2}$ and a side length $L$. Periodic boundaries are used throughout.

Figure 1. Lyapunov spectrum of a dense two-dimensional fluid consisting of $N=100$ particles at a density $\rho=0.7$ and a temperature $T=0.75$. The label WCA refers to a smooth Weeks-Chandler-Anderson interaction potential, whereas HD is for the hard disk system.

As an example we compare in Fig. 1 the Lyapunov spectrum of a WCA fluid with $N=100$ particles to an analogous spectrum for a hard disk system at the same temperature $(T=0.75)$ and density $(\rho=0.7)$. A renormalized index $l / 2 N$ is used on the abscissa. It is surprising that the two spectra differ so much in shape and in magnitude. The difference persists in the thermodynamic limit. The step-like structure of the hard disk spectrum for $l / 2 N$ close to 1 is an indication of a coherent wave-like shape of the associated perturbation. We defer the discussion of the so-called Lyapunov modes to Section 3.

\section{$2 \quad$ Fluctuating local exponents}

We infer from Equ. (1) that the Lyapunov exponents are time averages over an (infinitely) long trajectory and are global properties of the system. This time average can be written as

$$
\lambda_{l}=\lim _{\tau \rightarrow \infty} \int_{0}^{\tau} \lambda^{\prime}(\boldsymbol{\Gamma}(t)) d t \equiv\left\langle\lambda^{\prime}{ }_{l}\right\rangle,
$$

iwhere the (implicitely) time-dependent function $\lambda_{l}^{\prime}(\boldsymbol{\Gamma}(t))$ depends on the state $\boldsymbol{\Gamma}(t)$ in phase space the system occupies at time $t$. Thus, $\lambda_{l}^{\prime}(\boldsymbol{\Gamma})$ is called a local Lyapunov exponent. It may be estimated from

$$
\lambda^{\prime}{ }_{l}(\boldsymbol{\Gamma}(t))=\frac{1}{\Delta t_{G S}} \ln \frac{\mid \delta \boldsymbol{\Gamma}_{l}\left(\boldsymbol{\Gamma}\left(t+\Delta t_{G S}\right) \mid\right.}{\mid \delta \boldsymbol{\Gamma}_{l}(\boldsymbol{\Gamma}(t) \mid},
$$

where $t$ and $t+\Delta t_{G S}$ refer to times immediately after consecutive Gram-Schmidt re-orthonormalization steps. Its time average, denoted by $\angle \cdots\rangle$, along a trajectory gives the global exponent $\lambda_{l}$. The local exponents fluctuate considerably along a trajectory. This is demonstrated in Fig. ??, where we have plotted the second moment $\left\langle\lambda_{l}^{\prime 2}\right\rangle$ as a function of the Lyapunov index $1 \leq l \leq 4 N$ for a system of 16 particles, both for the WCA and HD particle interactions. $l=1$ refers to the maximum exponent, and $l=64$ to the most negative exponent. The points for $30 \leq l \leq 35$ correspond to the 6 vanishing exponents and are not shown. We infer from this figure that for the particles interacting with the smooth WCA potential the fluctuations of the local exponents, whose average give rise to global exponents approaching zero for $l \rightarrow 2 N$. For the hard disk system, however, the relative improtance of the fluctuations also becomes minimal in this limit. We shall return to this point in Section 3. 
We note that the computation of the second moments $\left\langle\lambda_{l}^{\prime 2}\right\rangle$ for the hard disk system requires some care. Due to the hard core collisions they depend strongly on $\Delta t_{G S}$ for small $\Delta t_{G S}$. The mean square deviations, $\left\langle\lambda^{\prime 2}\right\rangle-\left\langle\lambda^{\prime}{ }_{l}\right\rangle^{2}$, vary with $1 / \Delta t_{G S}$ for small $\Delta t_{G S}$, as is demonstrated in Fig. 3 for the maximum local exponent. However, the shape of the fluctuation spectrum is hardly affected by the size of the renormalization interval.

\section{The maximum exponent}

The maximum Lyapunov exponent is the rate constant for the fastest growth of phase space perturbations in a system. There is strong numerical evidence for the existence of the thermodynamic limit $\{N \rightarrow \infty, \rho=N / V$ constant $\}$ for $\lambda_{1}$ and, hence, for the whole spectrum. Furthermore, the associated perturbation is strongly localized in space. This may be demonstrated by projecting the tangent vector $\delta \boldsymbol{\Gamma}_{1}$ onto the subspaces spanned by the perturbation components contributed by the individual particles. The squared norm of this projection, $\delta_{i}^{2}(t) \equiv\left(\delta \mathbf{r}_{i}\right)_{l}^{2}+\left(\delta \mathbf{p}_{i}\right)_{l}^{2}$, indicates how active a particle $i$ is engaged in the growth process of the pertubation associated with $\lambda_{1}$. In Fig. $4 \delta_{i}^{2}(t)$ is plotted along the vertical for all particles of a hard disk system at the respective positions $\left(x_{i}, y_{i}\right)$ of the disks in space, and the ensuing surface is interpolated over a periodic grid covering the simulation box. A strong localization of the active particles is observed at any instant of time. Similar, albeit slightly broader peaks are observed for the WCA system.

This localization is a consequence of two mechanisms: firstly, after a collision the delta-vector components of two colliding molecules are linear functions of their pre-collision values and have only a chance of further growth if their values before the collision were already far above average. Secondly, each renormalization step tends to reduce the (already small) components of the other non-colliding particles even further. Thus, the competition for maximum growth of tangent vector components favors the collision pair with the largest components.

The localization also persists in the thermodynamic limit. To show this we follow Milanović et al. [8], square all $4 N$ components of the perturbation vector $\delta \boldsymbol{\Gamma}_{1}$ and order the squared components $\left[\delta \boldsymbol{\Gamma}_{1}\right]_{j}^{2} ; j=1, \ldots, 4 N$ according to size. By adding them up, starting with the largest, we determine the smallest number of terms, $A \equiv 4 N C_{1, \Theta}$, required for the sum to exceed a threshold $\Theta$. Then, $C_{1, \Theta}=A / 4 N$ may be taken as a relative measure for the number of components actively contributing to $\lambda_{1}$ :

$$
\Theta \leq\left\langle\sum_{s=1}^{4 N C_{1, \Theta}}\left[\delta \boldsymbol{\Gamma}_{1}\right]_{s}^{2}\right\rangle, \quad\left[\delta \boldsymbol{\Gamma}_{1}\right]_{i}^{2} \geq\left[\delta \boldsymbol{\Gamma}_{1}\right]_{j}^{2} \text { for } i<j .
$$

Here, $\langle\cdots\rangle$ implies a time average. Obviously, $C_{1,1}=1$. In Fig. $5 C_{1, \Theta}$ is shown for $\Theta=0.98$ as a function of the particle number $N$, both for the WCA fluid and for the hard disk system. It converges to zero if our data are extrapolated to the 
thermodynamic limit, $N \rightarrow \infty$. This supports our assertion that in an infinite system only a vanishing part of the tangent-vector components (and, hence, of the particles) contributes significantly to the maximum Lyapunov exponent at any instant of time.

\section{Lyapunov modes}

We have mentioned already the appearance of a step-like structure in the Lyapunov sepctrum of the hard disk system for the positive exponents closest to zero. They are a consequence of coherent wave-like spatial patterns generated by the perturbation vector components associated with the individual particles. In Fig. 6 this is visualized by plotting the perturbations in the $x$ (bottom surface) and $y$ direction (top surface), $\left\{\delta x_{i}, i=1, \ldots, N\right\}$ and $\left\{\delta y_{i}, i=1, \ldots, N\right\}$, respectively, along the vertical direction at the instantaneous particle positions $\left(x_{i}, y_{i}\right)$ of all particles $i$. This figure depicts a transversal Lyapunov mode, for which the perturbation is parpendicular to the wave vector, for a hard disk system consisting of $N=1024$ particles and for a perturbation vector associated with the smallest positive exponent $\lambda_{2045}$. An analogous plot for $\delta p_{x}$ and $\delta p_{y}$ $l=2045$ is identical to that of $\delta_{x}$ and $\delta_{y}$ in Fig. 6, with the same phase for the waves. This is a consequence of the fact that the perturbations are solutions first-order differential equation instead of second. Furthermore, the exponents for $2042 \leq l \leq 2045$ are equal. The four-fold degeneracy of non-propagating transversal modes, and an analogous eight-fold degeneracy of propagating longitudinal modes, are responsible for a complicated step structure for $l$ close to $2 N$, which has been studied in detail in Refs. XXXXX.

The wave length of the modes and the value of the corresponding exponents are determined by the linear extension $L$ of the simulation box. There is a kind of linear dispersion relation [10] according to which the smallest positive exponent is proprtional to $1 / L$. This assures that for a simulation box with aspect ratio 1 there is no positive lower bound for the positive exponents of hard disk systems in the thermodynamic limit.

So far, our discussion of modes is only for the hard disk fluid. In spite of a considerable computational effort we have not yet been able to indentify modes for two-dimensional fluid systems with a soft interaction potential such as WCA or similar potentials. The reason for this surprising fact seems to be the very strong fluctions of the local exponents as discussed in Section 2. The fluctuations obscure any mode in the system in spite of considerable averaging and make a positive identification very difficult. Three-dimensional systems are just beyond computational feasibility at present, although the use of parallel machines may change this scenario soon.

We are grateful to Christoph Dellago, Robin Hirschl, Bill Hoover, and Ljubo Milanović for many illuminating discussions. This work was supported by the Austrian Fonds zur Förderung der wissenschaftlichen Forschung, grants P11428PHY and P15348-PHY. 


\section{References}

1. Posch, H. A., and Hoover, Wm. G.: Equilibrium and nonequilibrium Lyapunov spectra for dense fluids and solids. Phys Rev. A 39 (1989) 2175-2188

2. Gaspard, P.: Chaos, Scattering, and Statistical Mechanics, (Cambridge University Press, 1998).

3. Hoover, Wm. G.: Computational Statistical Mechanics, (Elsevier, New York, 1999)

4. Dorfman, J.R.: An Introduction to Chaos in Nonequilibrium Statistical Mechanics, (Cambridge University Press, 1999)

5. Ruelle, D.: J. Stat. Phys. 95 (1999) 393

6. Benettin, G., Galgani, L., Giorgilli, A., and Strelcyn, J. M.: Meccanica 15 (1980) 9

7. Shimada, I., and Nagashima, T.: Proc. Theor. Phys. 61 (1979) 1605

8. Milanović, Lj., and Posch, H. A.: Localized and delocalized modes in the tangentspace dynamcs of planar hard dumbbell fluids. J. Molec. Liquids (2002), in press

9. Dellago, Ch., Posch, P. H., and Hoover, Wm. G.: Phys. Rev. E 53 (1996) 1485

10. 